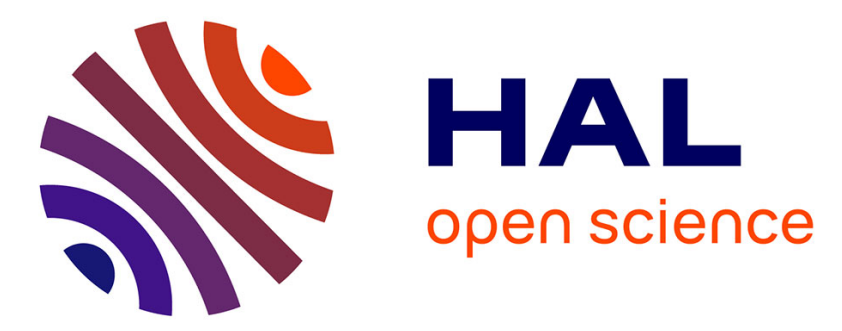

\title{
Prédiction des paramètres de la valeur alimentaire des repousses de la végétation naturelle de savanne de la zone tropicale humide par la technique de Spectrométrie dans le Proche InfraRouge
}

\author{
Ph Lecomte, R Agneessens, Ph Hellemans, P Dardenne, A Buldgen
}

\section{To cite this version:}

Ph Lecomte, R Agneessens, Ph Hellemans, P Dardenne, A Buldgen. Prédiction des paramètres de la valeur alimentaire des repousses de la végétation naturelle de savanne de la zone tropicale humide par la technique de Spectrométrie dans le Proche InfraRouge. Annales de zootechnie, 1996, 45 (Suppl1), pp.53-53. hal-00889585

\section{HAL Id: hal-00889585 https://hal.science/hal-00889585}

Submitted on 1 Jan 1996

HAL is a multi-disciplinary open access archive for the deposit and dissemination of scientific research documents, whether they are published or not. The documents may come from teaching and research institutions in France or abroad, or from public or private research centers.
L'archive ouverte pluridisciplinaire HAL, est destinée au dépôt et à la diffusion de documents scientifiques de niveau recherche, publiés ou non, émanant des établissements d'enseignement et de recherche français ou étrangers, des laboratoires publics ou privés. 


\title{
Prédiction des paramètres de la valeur alimentaire des repousses de la végétation naturelle de savanne de la zone tropicale humide par la technique de Spectrométrie dans le Proche InfraRouge
}

\author{
Ph Lecomte 1, R Agneessens 1, Ph Hellemans 2, P Dardenne 1, A Buldgen 2 \\ ${ }^{1}$ CRAG, Station de Haute Belgique, B6800 Libramon; 2FSAG, UER Zootechnie, 85030 Gembloux, Belgique
}

L'établissement de la valeur alimentaire d'un fourrage nécessite la détermination de paramètres portant sur le $\%$ de constituants, la teneur en énergie et la digestibilité. Pour qualifier 462 échantillons de repousse et biomasse collectés dans le cadre d'une étude sur les végétations de savane naturelle en région tropicale humide (Ranch de l'Adélé, Togo) nous avons eu recours à l'établissement de calibrages dans la technique de spectrométrie dans le proche infrarouge (SPIR).

Pour chacun des échantillons séchés $\left(65^{\circ} \mathrm{C}\right)$ et broyés (Cyclotec, 1,0 $\mathrm{mm}$ ) le spectre de réflexion proche infrarouge a été mesuré entre 1100 et $2500 \mathrm{~nm}$ à l'aide d'un monochromateur NIR System 5000 et traité à l'aide du logiciel ISI (InfraSoft International). La démarche a tout d'abord consisté à sélectionner un sousensemble représentatif. La technique consiste à rechercher la plus petite distance $\mathrm{H}$ entre 2 spectres et à éliminer l'un des deux. Par itérations successives on obtient un nombre d'échantillons, fixé par l'opérateur, occupant de la façon la plus homogène possible l'espace spectral engendré par la population initiale. Les paramètres déterminés sur chacun des échantillons sélectionnés sont : les cendres totales (CT), l'énergie brute (EB), la cellulose brute (CELL), les fractions fibreuses selon Van Soest (NDF), (ADF), (ADL), la matière protéique totale (MPT). La digestibilité a été estimée selon la technique au jus de rumen (ttMS) (Tilley et Terry, 1963, J Brit Grassl Soc, 18, 104-111) et selon la technique enzymatique de solubilisation à la pepsine-cellulase (casedMO) (Aufrère, 1982, Ann Zootech, 31, 111-130). La technique Partial Least Squares (Martens et al, 1982, Proc World Cereal Bread Congr, 7th, Elsevier, Amsterdam, 607-647) a été utilisée pour établir

$\begin{array}{lcc}\text { Critères } & n & \text { Min - Max } \\ \text { CT (\%MS) } & 76 & 4,15-11,68 \\ \text { MPT (\%MS) } & 78 & 2,02-11,22 \\ \text { CELL (\%MS) } & 78 & 27,97-43,28 \\ \text { NDF (\%MS) } & 78 & 59,30-77,47 \\ \text { ADF (\%MS) } & 78 & 32,91-51,10 \\ \text { ADL (\%MS) } & 78 & 3,61-9,41 \\ \text { CasedMO (\%MO) } & 77 & 17,82-55,66 \\ \text { tIMS (\%MS) } & 49 & 26,12-57,02 \\ \text { EB (Mj/kgMS) } & 48 & 17,03-18,27\end{array}$

le calibrage de chacun des paramètres. La validation des équations, effectuée à chaque étape pour un nombre croissant de termes, consiste à choisir systématiquement $3 / 4$ des échantillons du sous ensemble, sur lesquels un modèle est développé, celui-ci est ensuite appliqué au 1/4 des échantillons restants, et aboutit à un écart résiduel de prédiction sur des échantillons indépendants. La moyenne quadratique des écarts donne une erreur de validation croisée dont le minimum fixe le nombre de termes à retenir dans le modèle.

Les modèles sont caractérisés (tableau) par les coefficients de détermination en calibration $R^{2} c$ et en validation croisée $R^{2} c v$ exprimant dans les deux cas la part de variation expliquée par la régression ainsi que par les écarts types résiduels en calibration $\left(S E_{C}\right)$ et en validation croisée $\left(S E_{C V}\right)$ qui expriment la variabilité de l'erreur commise par le modèle sur les échantillons pris en calibration, et en validation, sur ceux n'ayant pas participé à la calibration.

Les modèles sont d'une façon générale très précis, ils atteignent des valeurs comparables à celles obtenues avec des fourrages tempérés. L'énergie brute est un paramètre intéressant à calibrer. Sa précision $(0,16 \mathrm{Mj} / \mathrm{kg} \mathrm{MS})$ est de 2 fois celle de la mesure de référence à la bombe. Les différents calibrages que l'on a pu établir montrent l'intérêt de la SPIR pour déterminer de façon rapide et peu coûteuse les paramètres de qualité de populations importantes. La technique SPIR est très reproductible dans le temps et dans l'espace. Elle constitue une alternative interessante à l'équipement de routine ou de recherche dans des régions où les intrants necessaires à l'analyse classique sont difficilement accessibles.

\begin{tabular}{|c|c|c|c|c|}
\hline Moy. & $\mathrm{SE}_{\mathrm{C}}$ & $\mathrm{R}^{2} \mathrm{c}$ & $S E_{c y}$ & $\mathrm{R}^{2} \mathrm{cV}$ \\
\hline 7,95 & 0,80 & 0,77 & 0,95 & 0,69 \\
\hline 6,44 & 0,43 & 0,97 & 0,48 & 0,96 \\
\hline 34,61 & 0,74 & 0,96 & 0,87 & 0,95 \\
\hline 66,81 & $\uparrow, 12$ & 0,94 & 1,27 & 0,93 \\
\hline 40,11 & 0,99 & 0,96 & 1,21 & 0,94 \\
\hline 5,55 & 0,41 & 0,93 & 0,59 & 0,85 \\
\hline 38,76 & 2,23 & 0,95 & 2,64 & 0,93 \\
\hline 45,64 & 1,41 & 0,97 & 2,09 & 0,94 \\
\hline 17,60 & 0,12 & 0,86 & 0,16 & 0,77 \\
\hline
\end{tabular}

